\title{
Designing and Evaluating Mobile Phone-Based Interaction with Public Displays
}

Corina Sas

Computing Department

Lancaster University Lancaster, LA1 4WA, UK

c.sas@lancaster.ac.uk

Alan Dix

Computing Department

Lancaster University

Lancaster, LA1 4WA, UK

alan@hcibook.com

\section{Abstract}

This paper outlines the

rationale for the

workshop topic and

offers an overview of its

objectives.

\section{Keywords}

Mobile phones, public

displays, interaction

design, evaluation.

\section{ACM Classification}

Keywords

H. 5 Information

interfaces and

presentation: H.5.2

User Interfaces and

H.5.3 Group and

Organization Interfaces.

\begin{abstract}
Introduction
Personal devices including mobile phones, PDAs or MP3 players are becoming increasingly pervasive. Alongside this, large displays are becoming a common site in public places: offices, airports, shops and town centers. On the one hand, these technologies are being studied separately in both mobile HCI and pervasive computing fields although work starting to appear that bridges the two. On the other hand, there are inherent limitations characterizing each of these technologies such as small form-factor of mobile phones or insufficiently matured techniques for supporting interaction with public displays. The latter is increasingly important since despite their increased ubiquity, public displays have traditionally been location configured broadcasts whose displayed content usually ignores the presence and interests of those watching it.

From such limitations a strand of research has emerged into the symbiotic relationship between these technologies, where the strengths of each match the limitations of the other, e.g. the interactive capabilities of the mobile phone compliment the large form-factor of the public display [6]. This symbiotic relationship between mobile phones and public displays (where personal, small and private meets public, large and
\end{abstract}


shared) can also be used to explore additional challenges of public displays.

\section{Challenges}

As Agamanolis explains [1], half the battle in designing an interactive public display is how to invite and maintain interaction even after the novelty factor wears off. For instance, the leverage of users' familiarity with mobile phones and of their content privacy could be exploited to engage people and overcome their initial resistance to interact with public displays [5].

Other challenges of designing interactive public displays relate to the control of the interaction since both the content and the pace at which it responds is no longer own by an individual user but belongs to other users, context, or broadcast streams.

Finally, there are specific evaluation challenges of designing interactive public displays beyond those of the individual technologies; for example, long-term use by occasional users. Specifically, the design and development of new interaction techniques is currently hindered by the lack of proper evaluation methodology and methods. The complexity of interaction is due to the interrelation between the two types of technology added to the multiple people, activities and contexts [7]. Therefore, the situational aspect of this type of interaction challenges the effectiveness of traditionally design and evaluation methods from data gathering to data interpretation [3]. Situated experimentation offers a way to address the limitations of laboratory studies while new research tools able to capture the richness of real data (such as Momento [3]) still need to be developed.
Given the infancy of this field, the focus on the development of interaction techniques with public displays has perhaps focused more on technical aspects and less on social and interactional ones. Rigorous evaluation and systematic comparisons between such techniques is both difficult and rare.

\section{Interaction techniques}

The mobile phone-based interaction techniques that have been developed so far fall into three main classes. In some the personal device effectively acts as an extended input device for text or pointing. In others the phone is used to upload and download media using standard content and protocols. The third class is where the mobile phone is a more integral part of the interaction, typically using the display of the device in concert with the public display. In addition phones may be used to identify users and displays to one another.

The mobile phones used as pointing devices often exploit the properties of camera based interaction which has the benefit of offering direct manipulation of the pointer position and even continuous visual feedback [4]. In this way, the interaction techniques range from exploring the natural movement of the camera-phone along the desired direction of the cursor motion, e.g. sweep; to the use of visual codes, e.g. point \& shoot [2].

Where the phone is a more integral part of the interaction we get additional issues due to the separation of attention between small screen and large screen. However, also we get opportunities for example allowing more private information relating to interactions to be displayed on the private device. This also creates new architectural challenges as the 
available devices constantly change and information may have to move between larger and smaller formfactors.

Finally public displays are, by definition, public and so individual interactions affect what is seen by bystanders. So we get issues of what is acceptable to be displayed to others: is it offensive to others, is it enjoyable for them? This also impacts architecture and infrastructure: how does the display know which devices to trust, or which sources of information are acceptable?

\section{Workshop Objectives}

The proposed workshop will serve as a forum for exchanging ideas on designing and evaluating innovative interaction techniques between mobile phones and public displays. It aims to address the question of how could mobile phones be used to interact with public displays. The workshop also aims to contribute to the field through:

- the development of a theoretical framework able to explain and predict user's mobile phone-based interaction with public displays.

- the development of a methodological framework able to support the investigation and evaluation of user's mobile phone-based interaction with public displays.

- the development of a taxonomy of interaction techniques together with a set of guidelines for their usage appropriateness which will capture application-, task-, time-, and locationdependency.

- the development of requirements for the future infrastructure and architecture of these systems.
Research Questions

The workshop's aim and objectives will be addressed through the following questions:

- Which are the most important user's aspects when designing techniques for mobile phone-based interaction with public displays?

- How could designers account for privacy issues related to displaying personally relevant information on public displays, e.g. does it happen and in which conditions?

- How could designers account also for the acceptability of information or media to bystanders of public displays while still allowing personal interaction?

- Which design methodology could be used to generate and test ideas for simultaneously explore the strengths and overcome the limitations of each technology?

- How could designers account for the cognitive demands and attentional shift that occur from simultaneous engagement with both technologies?

- How the situated aspects of interacting with public displays impact on the quality of user experience?

- Which are the problems of applying traditional evaluation methods for evaluating innovative interaction techniques with public displays?

- Which are the criteria for assessing interaction techniques and how could they be measured, e.g. performance, quality of experience?

- What kinds of underlying system infrastructure are required for developing, deploying and maintaining such systems?

- Which are the ethical issues regarding the use studies for mobile phone-public display interaction? 
Scope

The workshop accepts original contributions relating (but not limited) to:

- Designing innovative mobile phone-based interaction techniques with public displays.

- Designing for engagement with public displays.

- Design methodology and guidelines for interaction techniques.

- Human factors in mobile-phone based interaction techniques with public displays.

- Methods and tools for evaluating interaction techniques.

- Multi-modal interaction techniques.

- Fun and game related aspects pertaining to innovative interaction techniques.

- Social aspects related to these interaction techniques: sharing, privacy, ownership, competition-cooperation, engagement, fun.

\section{References}

[1] Agamanolis, S. New Technologies for Human

Connectedness, ACM Interactions, 12, 4 (2005), 33-37.

[2] Ballagas, R., Rohs, M. and Sheridan, J.G. Sweep

and point and shoot: Phonecam-based interactions for large public displays. In Extended Abstracts CHI 2005, ACM Press (2005), 1200-1203

[3] Carter, S., Mankoff, J., Heer, J. Momento: Support for situated ubicomp experimentation. In Proc. CHI 2007, ACM Press (2007), 125-134.

[4] Jiang, H., Ofek, E, Moraveji, N. and Shi, Y. Direct pointer: direct manipulation for large-display

interaction using handheld cameras. In Extended Abstracts CHI 2006, ACM Press (2006), 1107-1110.

[5] Mitchell, K., Race, N.J.P., Suggitt, M. iCapture: Facilitating Spontaneous User-Interaction with

Pervasive Displays using Smart Devices, in Proc. Mobile Devices as Pervasive User Interfaces and Interaction Devices (PERMID), (2006).

[6] Raghunath, M.T., Ravi, N., Rosu, M.C., Narayanaswami, C. Inverted browser: A novel approach towards display symbiosis. In Proc. of PerCom 2006 (2006), 71-76.

[7] Zhang, D. and Adipat, B. Challenges, methodologies, and issues in the usability testing of mobile applications. IJHCI 18, 3 (2005), 293-308. 